\title{
Why and when to prefer botulinum toxin injection in childhood strabismus?
}

\author{
Kadriye Erkan-Turan, Hande Taylan-Şekeroğlu, Abdullah Ağın, Ali Şefik Sanaç \\ Department of Ophthalmology, Hacettepe University Faculty of Medicine, Ankara, Turkey. \\ E-mail: kadriyerkan@gmail.com \\ Received: 28th January 2017, Accepted: 7th May 2017
}

SUMMARY: Erkan-Turan K, Taylan-Şekeroğlu H, Ağın A, Sanaç AŞ. Why and when to prefer botulinum toxin injection in childhood strabismus? Turk J Pediatr 2017; 59: 684-687.

The aim of this study was to investigate the indications and outcomes of botulinum toxin injection in children with strabismus. The medical records of children who underwent botulinum toxin injection were reviewed. Eighteen patients ( 13 boys, 5 girls) with a mean age of $9.08 \pm 5.93$ (6 months to 17 years) years were enrolled in the study. The main indication and diagnosis, initial and final deviation at primary position and the presence of abnormal head position were all evaluated. The most common diagnosis was sixth nerve palsy ( 7 patients) followed by Duane's syndrome ( 4 patients), acquired esotropia ( 2 patients), acquired exotropia ( 2 patients), consecutive exotropia (2 patients) and sensory strabismus (1 patient). The leading indications for botulinum toxin injections were the presence of abnormal head position, diplopia and ocular misalignment at primary position. All patients received monocular injection. Fourteen patients had one, 4 patients had more than one injection. The mean follow-up period was $2.78 \pm 0.94$ months for the first post-injection visit and $21.64 \pm 15.23$ months for the last visit. Five patients underwent strabismus surgery due to inadequate response to injection. No complication related to injection was observed. Botulinum toxin injection may be preferred in pediatric age group particularly in case of extraocular muscle palsy, diplopia and concomitant deviation either to provide ocular alignment prior to surgery or to prevent the detrimental effect of diplopia on binocularity. The intervention seems to be safe and repeatable in children even though surgery is still inevitable in particular cases.

Key words: botulinum toxin, children, injection, strabismus.

Botulinum toxin type A (BTA) has been studied since 1979 for selective weakening of extraocular muscles. ${ }^{1}$ Botulinum toxin has been used for diagnosis and treatment of different types of strabismus. ${ }^{2}$ Uses of botulinum toxin for diagnostic purposes in strabismus include evaluation of postoperative diplopia, preoperative fusion presence, differential diagnosis of sixth nerve palsy, prediction of the surgical results for incomitant deviations and a possible slipped muscle following surgery. ${ }^{2}$ Botulinum toxin has been also used for therapeutic purposes such as fusion restoration in patients with decompensating deviations or sixth nerve palsy, rehabilitation of surgical overcorrections and undercorrections, ocular alignment and improvement of visual acuity in patients with acquired nystagmus. ${ }^{2}$

In the present study, we aimed to report the outcomes of botulinum toxin injection in children with strabismus by going through discussion of clinical features of the patients and main indications of botulinum in pediatric age group.

\section{Material and Methods}

The clinical records of the patients who underwent botulinum toxin injection for strabismus between 2013 and 2016 were 
reviewed upon approval of the Institutional Review Board. The authors adhered the tenets of the Declaration of Helsinki. Information regarding alternative approaches, namely prismatic correction, strabismus surgery, and possible outcomes, particularly limitation in different gaze positions, ptosis were given to all patients and their parents. The patients who were keen on botulinum toxin injection procedure and had at least 2 months of followup post-injection were enrolled in the study. Exclusion criteria were as follows: previous ocular surgery except for strabismus surgery, and previous botulinum toxin injection.

The medical files of the patients were retrospectively reviewed. Detailed medical history, age at surgery, gender, etiology strabismus, visual acuity, type of deviation, anterior segment and fundus examination findings were all recorded. Visual acuity was assessed using a Snellen chart in older children and LEA symbols in younger children. The angle of pre-injection and post-injection deviations were assessed by Hirschberg test, Krimsky test or the alternate prism cover test (in prism diopters, PD) when possible. Measurements were taken at distance and near when possible, as well. Sensory fusion was evaluated with Worth 4-Dot Test and stereopsis was assessed with Titmus test. Presence of abnormal head position was evaluated.

The patients included in the study received at least one botulinum toxin injection (Botox ${ }^{\circledR}$, Allergan Pharmaceuticals, USA) in a surgical room under inhalational anesthesia. Botulinum was reconstituted with sterile, non-preserved normal saline ( $0.9 \%$ sodium chloride). The dose of botulinum toxin was 4 units and the injection was performed under direct observation of the extraocular muscle. Injections were performed with a 27-gauge needle on an insulin syringe without EMG guidance. Area of botulinum toxin injection was based on anatomical landmarks.

\section{Statistical analysis}

Statistical analyses were performed by IBM SPSS for Windows Version 22.0 statistical package. Continuous variables presented as mean \pm standard deviation (min - max). Categorical variables were summarized as frequencies and percentages.

\section{Results}

Eighteen patients (13 boys, 5 girls) with a mean age of $9.08 \pm 5.93$ ( 6 months to 17 years) years met the criteria for inclusion among the patients who underwent botulinum toxin injection between 2013 and 2016. The most common diagnosis was sixth nerve palsy (7 patients, $38.8 \%$ ) followed by Duane's syndrome (4 patients, $22.2 \%$ ). Other diagnoses were consecutive exotropia, acquired esotropiaexotropia and sensory strabismus. The leading indications for botulinum toxin injections were the presence of abnormal head position, diplopia and ocular misalignment at primary position. Mean time between first examination and injection was $1.47 \pm 0.65$ months (15 days to 3 months). All patients received monocular injection. Fourteen patients had one, 4 patients had more than one injection. The mean follow-up period was 2.78 months (2 - 4 months) for the first post-injection visit and 21.64 months (11 - 49 months) for the last visit. Two patients dropped out from the long-term follow-up. The angle of deviation decreased after injection in patients whereas five patients needed to undergo strabismus surgery due to inadequate response to injection. No complication related to injection was observed. Clinical characteristics of the patients were given in detail in Table I.

\section{Discussion}

Botulinum toxin injection to extraocular muscles is a well-established alternative option in the treatment of adult strabismus whereas its use in children is limited and less studied. ${ }^{2}$ Majority of published literature regarding botulinum toxin injection in strabismus consists of retrospective studies, cohort studies or case reviews. ${ }^{2}$

Jarrin et al. ${ }^{3}$ investigated the effect of botulinum toxin injection in 11 children with a mean age of 4.42 years and found that it was effective in decreasing the angle of deviation and even further it may provide improvement of stereoacuity with a lower number of injection compared to adults. Hung et al. ${ }^{4}$ reviewed the outcomes of patients with traumatic sixth nerve palsy who received botulinum toxin injection within 3 months of injury and suggested that botulinum toxin may be helpful in the improvement of the palsy particularly in patients with severe injury. 


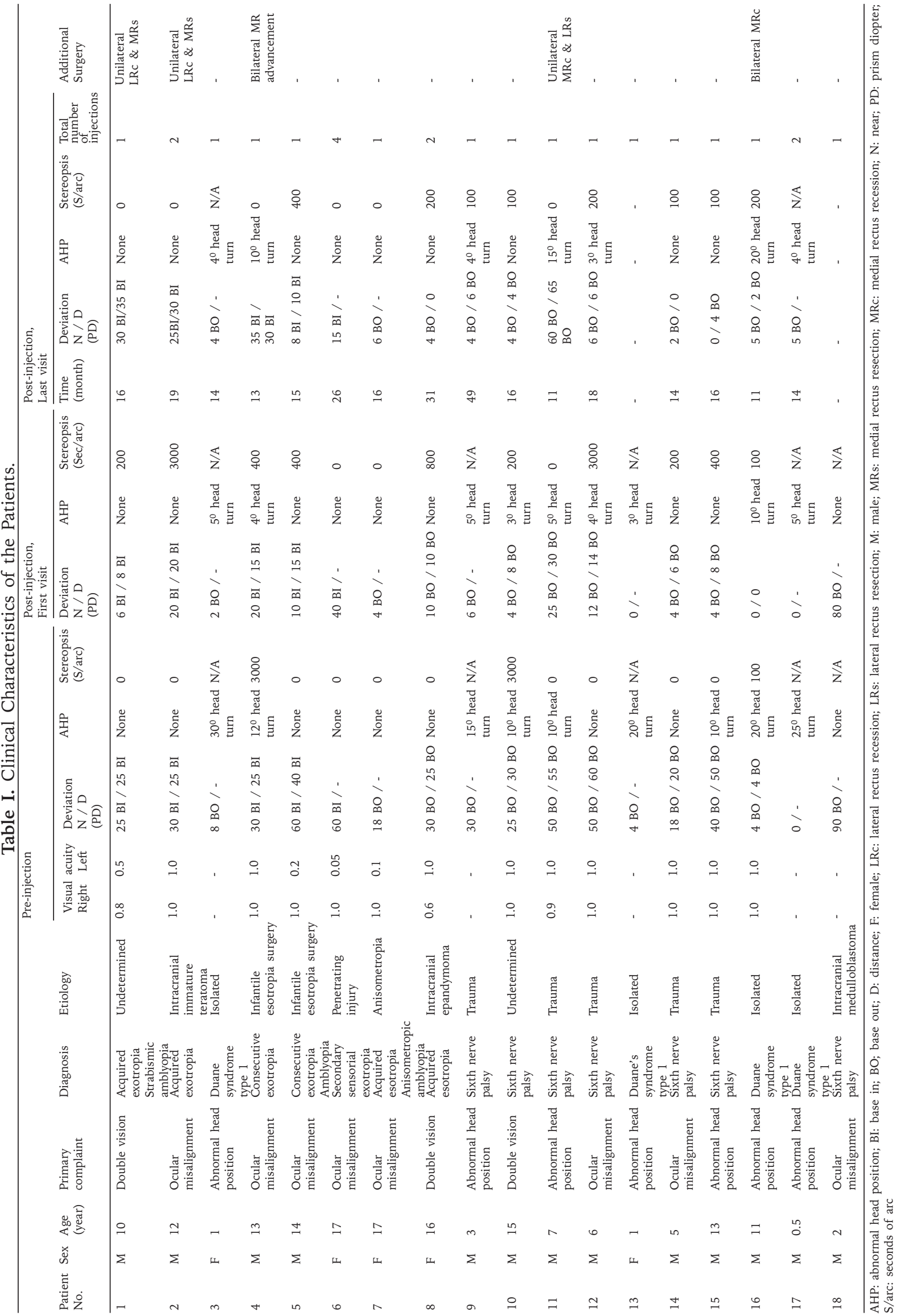


In the present study, 4 patients had botulinum injection for the correction of abnormal head position due to Duane's syndrome. Maya et al. ${ }^{5}$ performed botulinum injection in children with Duane's syndrome and found that it may be considered as an alternative option to surgery when the primary indications are esotropia and face turn. Dawson et al. ${ }^{6}$ used botulinum toxin to simulate the surgical outcome in patients with Duane's syndrome and showed that botulinum toxin not only decreased the angle of deviation but also offered long-term maintenance of good ocular alignment.

Dawson et al. ${ }^{7}$ reported the use of botulinum toxin in children with acute onset comitant esotropia and said that binocularity and good ocular alignment may be achievable upon injection. Wan et al. ${ }^{8}$ compared surgery and chemodenervation in children with acute comitant esotropia and suggested that botulinum is cost-effective and had similar success rate to surgery. Botulinum toxin may be also used in residual, consecutive and secondary deviations. ${ }^{9-11}$

In our series, satisfactory results were obtained in two-thirds of the children treated with one or more botulinum toxin injections in long-term follow-up. The angle of abnormal head position and deviation in primary position decreased, diplopia resolved and furthermore binocularity was gained in some of the patients.

Botulinum toxin injection may be preferred in pediatric age group particularly with extraocular muscle palsy, diplopia and concomitant deviation either to provide ocular alignment prior to surgery or to prevent the detrimental effect of preoperative or postoperative diplopia by restoring binocularity. Botulinum toxin injection seems to be safe and repeatable in children even though in particular cases surgery is still an irreplaceable treatment option. However, as being less invasive compared to surgery, botulinum toxin seems to be preferable when prompt intervention is needed such as in case of risk of binocularity loss, the outcome is unpredictable due to complexity of the deviation or when the surgery is inconvenient because of the risk of prolonged duration of general anesthesia.
In conclusion, botulinum toxin injection should be considered as a good alternative especially in young patients and in patients unsuitable for surgery due to accompanying systemic disorders.

\section{REFERENCES}

1. Sugano DM, Fernandez CL, Rehder JRCL. Botulinum toxin for strabismus correction. Rev Bras Oftalmol 2013; 72: 321-325.

2. Rowe FJ, Noonan CP. Botulinum toxin for the treatment of strabismus. Cochrane Database Syst Rev 2017; CD006499.

3. Jarrín E, Arranz Márquez E, Yebra González L, García Gil de Bernabé J. Botulinum toxin uses in strabismus: A review of the injections performed during one year in a general hospital. Arch Soc Esp Oftalmol 2016; 91: 114-119.

4. Hung HL, Kao LY, Sun MH. Botulinum toxin treatment for acute traumatic complete sixth nerve palsy. Eye (Lond) 2005; 19: 337-341.

5. Maya JF, de Liaño RG, Catalán MR, Rayward O. Botulinum toxin treatment in patients up to 3 years of age who have esotropic Duane retraction syndrome. Strabismus 2013; 21: 4-7.

6. Dawson EL, Maino A, Lee JP. Diagnostic use of botulinum toxin in patients with Duane syndrome. Strabismus 2010; 18: 21-23.

7. Dawson EL, Marshman WE, Adams GG. The role of botulinum toxin A in acute-onset esotropia. Ophthalmology 1999; 106: 1727-1730.

8. Wan MJ, Mantagos IS, Shah AS, Kazlas M, Hunter DG. Comparison of botulinum toxin with surgery for the treatment of acute-onset, comitant esotropia in children. Am J Ophthalmol 2017; 176: 33-39.

9. Lambert SR, Shainberg MJ. The efficacy of botulinum toxin treatment for children with a persistent esotropia following bilateral medial rectus recessions and lateral rectus resections. Am Orthopt J 2013; 63: 24-28.

10. Couser NL, Lambert SR. Botulinum toxin a treatment of consecutive esotropia in children. Strabismus 2012; 20: 158-161.

11. Dawson EL, Sainani A, Lee JP. Does botulinum toxin have a role in the treatment of secondary strabismus? Strabismus 2005; 13: 71-73. 